$63^{\text {ème }}$ Congrès de la SFCO, 02022 (2015)

DOI:10.1051/sfco/20156302022

(C) Owned by the authors, published by EDP Sciences, 2015

\title{
COMMUNICATION
}

\section{« Le DES de Chirurgie Orale : le bilan de la première promotion sortante »}

\author{
Devisse $\mathrm{M}^{*}$, Douilly G**, Bridonneau T***, Le Toux G*, Boisramé $\mathrm{S}^{*}$ \\ * CHRU UFR Odontologie Service d'Odontologie, 29200 Brest \\ ** CHRU Service d'Odontologie 1146 Avenue du Père Soulas, 34000 Montpellier \\ *** CHRU UF Chirurgie Buccale-Implantologie, Pôle de médecine et chirurgie bucco-dentaire, 1 place de \\ l'hôpital, 67000 Strasbourg
}

En 1995, c'est la création de l'internat en Odontologie.

En 2011, voit le jour l'internat d'Odontologie qualifiant pour trois spécialités : l'Orthopédie DentoFaciale, la Médecine Bucco-Dentaire et la Chirurgie Orale est formée.

En 2015, sont célébrés les 20 ans de l'internat en Odontologie et la sortie de la première promotion d'internes qualifiés en Chirurgie Orale.

Le Diplôme des Études Supérieures de Chirurgie Orale (DESCO) a été créé à la suite d'une demande de santé publique en lien avec les prévisions démographiques faisant apparaître un déficit de praticiens assurant les missions de ces chirurgiens oraux. Il remplace en 2011 le Diplôme des Études Supérieures de Chirurgie Buccale (DESCB) côté odontologie et le Diplôme des Études Supérieures de Stomatologie, côté médecine. En effet, le DESCO rassemble des praticiens médecins et chirurgiensdentistes, deux filières de formation, deux conseils de l'ordre.

Le DESCO se voit apporter des changements majeurs par rapport aux anciens DES, notamment, l'ouverture de la discipline d'odontologie aux internes venant de la filière médecine, et de la discipline médicale aux internes venant de la filière odontologiste.

Le rôle de l'interne en Chirurgie Orale est la prise en charge médico-chirurgicale des patients. Dans les services agréés, l'interne s'occupe de la chirurgie des tissus durs (avulsion simple ou complexe, exérèse de kyste, chirurgie pré-implantaire et implantaire, dégagement ortho-chirurgical) et des tissus mous (gingivoplasties, dermatologie buccale, lithiase salivaire). Spécifiquement, dans les services de Chirurgie Maxillo-Faciale, l'interne apprend à gérer la traumatologie des maxillaires, les chirurgies pré-implantaires étendues avec des prélèvements extra-buccaux. Il assiste en qualité d'aide opératoire à des chirurgies maxillo-faciales lourdes traumatiques ou cancéreuses, ou de chirurgies orthognathiques. Dans tous les services, l'interne a ses obligations de gestion des urgences et d'encadrement d'externes.

Selon les centres hospitaliers, le rôle de l'interne peut varier. Bien évidemment, comme dans toute spécialité médico-chirurgicale, des disparités sont à noter selon les régions concernant les notions et les techniques enseignées.

Malgré la légitimité au niveau santé publique de cette spécialité, des problèmes et des flous persistent quant à sa reconnaissance notamment par le conseil de l'ordre des médecins. De plus, le DESCO n'étant pas inscrit dans la directive européenne 2005/36, il n'y a pas d'équivalence européenne.

Des disparités existent entre les DESCO médecins et les DESCO dentaires, lors de leur formation et pour leur exercice futur.

This is an Open Access article distributed under the terms of the Creative Commons Attribution License 4.0, which permits unrestricted use, distribution, and reproduction in any medium, provided the original work is properly cited. 
Le but initial de ce diplôme a-t-il été atteint ?

La formation de l'interne DESCO est-elle supérieure à celle de l'interne de DES de stomatologie et de chirurgie buccale?

Le chirurgien oral prendra-t-il sa place au sein de la communauté chirurgicale ?

Quel est le ressenti des internes DESCO sortants ?

Nous tenterons de donner un début de réponse à ces questions au cours de notre exposé en s'appuyant sur les résultats d'un questionnaire de satisfaction rempli par les internes sortants.

Nom et adresse du conférencier

\section{Manon DEVISSE}

CHRU UFR Odontologie Service d'Odontologie

29200 Brest (France)

manon.dev@hotmail.fr 